\title{
Improving Undersampled MRI Reconstruction Using Non-Local Means
}

\author{
Ganesh Adluru ${ }^{1}$, Tolga Tasdizen ${ }^{2,3}$, Ross Whitaker ${ }^{2}$, Edward DiBella ${ }^{1}$ \\ ${ }^{1}$ UCAIR, Department of Radiology, ${ }^{2}$ SCI Institute, School of Computing, ${ }^{3}$ Electrical \& Computer \\ Engineering, University of Utah \\ \{gadluru,ed\}@ucair.med.utah.edu, \{tolga,whitaker\}@sci.utah.edu
}

\begin{abstract}
Obtaining high quality images in MR is desirable not only for accurate visual assessment but also for automatic processing to extract clinically relevant parameters. Filtering-based techniques are extremely useful for reducing artifacts caused due to undersampling of $k$-space (to reduce scan time). The recently proposed Non-Local Means (NLM) filtering method offers a promising means to denoise images. Compared to most previous approaches, NLM is based on a more realistic model of images, which results in little loss of information while removing the noise. Here we extend the NLM method for MR image reconstruction from undersampled $k$-space data. The method is applied on T1-weighted images of the breast and T2-weighted anatomical brain images. Results show that NLM offers a promising method that can be used for accelerating MR data acquisitions.
\end{abstract}

\section{Introduction}

Image quality and high signal to noise ratio (SNR) are always sought in MRI for better visual assessment and accurate quantification of clinical parameters. The inherent low signal level in MRI and the desire to acquire images rapidly motivates the use of postprocessing methods for obtaining better images. A number of filtering techniques have been proposed and have played a crucial role in improving image quality in cases when k-space data were fully sampled (for removing noise) as well as when undersampled (in order to reduce the scan time).

When k-space data are undersampled, artifacts arising due to violation of Nyquist criterion can also be resolved using filtering techniques. The UNFOLD method for dynamic imaging $[1,2]$ uses a temporal low pass filter to remove the side lobes at the Nyquist frequencies arising from interleaved undersampling. In order to obtain even higher acceleration factors the k-t BLAST method based on a Wiener filter approach was proposed [3]. The method uses training data to capture the spatio-temporal characteristics of dynamic images and designs a filter to effectively resolve the artifacts in the undersampled acquisition. More recently non- linear Total Variation (TV) filtering method [4] has been extended to remove the artifacts from undersampling. In this approach, instead of preserving fidelity to the entire image as in standard image denoising [4], data fidelity is preserved only at the sampled locations in $\mathrm{k}$-space while minimizing the $L_{1}$ norm of the gradients of the signal intensities. This method fits in the mathematical framework of compressed sensing, which suggests exact reconstructions of a sparse signal from incomplete Fourier data can be possible by minimizing its $L_{1}$ norm $[5,6]$. The method was applied to accelerating a number of dynamic and static MR imaging techniques [7-9]. However a limitation of the TV approach is that fine structures in images can be lost or have poor contrast [8].

Recently a Non-Local Means denoising method was proposed by Baudes et al [10], which is based on averaging similar pixels in the image by exploiting the natural redundancy in images. At the same time, an alternative approach based on a statistical framework, unsupervised information-theoretic adaptive filtering (UINTA), was introduced [11,12]. These methods produced state-of-the-art denoising results on a wide variety of naturally occurring images that have a lot of texture. NLM technique was adapted and applied to simulated and actual 2D/3D brain MR images [13, 14] and also extended to simultaneously use information from multi-component T1-weighted, T2-weighted and proton density images of the brain for denoising each individual image [15]. Unlike its previous MR applications for image denoising, here we extend the method to do MR image reconstruction from undersampled $\mathrm{k}$-space data. The approach is tested on in-vivo MR data of perfusion of breast and anatomical brain.

\section{Methods}

\subsection{Non-Local Means}

In the NLM algorithm, each pixel of interest $p$ in the given image $m$ is denoised by computing a weighted average of pixels that are not necessarily spatially local to $p$. The filtered image, $\widehat{m}$ is given by 


$$
\widehat{m}(p)=\frac{\sum_{q \in \operatorname{Win}(p)} w(p, q) m(q)}{\sum_{q \in \operatorname{Win}(p)} w(p, q)}
$$

where $\operatorname{Win}(p)$ is a spatial search window around pixel $p$ whose pixels are used for computing the weighted mean. The weights, $w(p, q)$ are computed based on the amount of similarity in spatial neighborhoods according to

$$
\begin{gathered}
w(p, q)=e^{\frac{-d(p, q)}{h^{2}}} ; \\
d(p, q)=G_{\sigma}\left\|m\left(N_{p}\right)-m\left(N_{q}\right)\right\|_{2}^{2}
\end{gathered}
$$

where $d(p, q)$ is the Gaussian weighted $L_{2}$ norm distance between the neighborhoods $N_{p}$ and $N_{q}$ around pixels $p$ and $q$ respectively within $\operatorname{Win}(p)$. $h$ is the filtering parameter which controls decay of the weights. In essence a pixel that is more similar to the pixel of interest in terms of spatial neighborhoods receives higher weight. In order to avoid overweighting of the pixel $p, w(p, p)$ is chosen as the maximum of the weights computed for other pixels, rather than 1 .

\subsection{MR image reconstruction}

When k-space data are undersampled, the resulting artifacts can be resolved basing on the above filter by minimizing the functional,

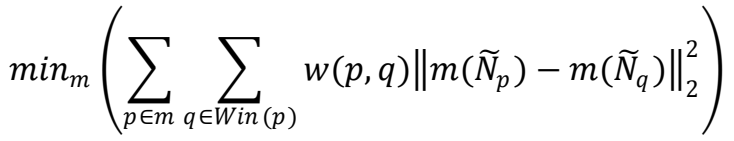

$$
\begin{aligned}
& \text { s.t. }\|E m-d\|_{2}^{2}<\varepsilon
\end{aligned}
$$

In the above expression, $E$ is the forward modeling operator for the image estimate $(m)$ which computes its Fourier transform at the k-space sampling locations to match the acquired data, $d . \widetilde{N}_{p}$ and $\widetilde{N}_{q}$ are neighborhoods around pixels $p$ and $q$ respectively that are not necessarily same as $N_{p}$ and $N_{q}$. The minimization term penalizes the weighted $L_{2}$ norm distance between neighborhoods in the images. This is in contrast to the TV term, which penalizes $L_{1}$ norm of the local intensity gradients. Motivation for using such a non-local term stems from the fact that images in general have redundancies in terms of neighborhoods rather than local/individual pixels. Image reconstruction is performed using an alternating minimization scheme in a POCS framework [16-18]. In each iteration, the complex image estimate is projected on to the data fidelity term and minimization of the neighborhood penalty term is performed. In order to simplify computation of the minimization term and its derivative, we make two assumptions ( $i$ ) $\widetilde{N}_{q}$ represents a $1 X 1$ neighborhood for pixel $q$ and (ii) only one sub-iteration of the penalty term is done with constant pre-determined weights. With these assumptions, minimization of the penalty functional is equivalent to applying the NLM filter in Eqn. (1) on $m$ as was shown in [19]. The above simplified framework favors a reconstructed image such that each pixel in it is similar to a larger neighborhood of pixels with Gaussian weighting while ensuring data consistency. The steps in the reconstruction algorithm are outlined below.

(i) Choose an initial guess: $m^{0}$

(ii) Project the current image estimate on-to the data consistency term: $m_{\text {data }}^{n}=m^{n}+E^{\prime}\left(d-E m^{n}\right), E^{\prime}$ is the adjoint operator of $E$

(iii) Compute weights $w(p, q)$ according to Eqn. (2) using $m_{\text {data }}^{n}$.

(iv) Minimize the neighborhood term by applying once NLM separately on the real and imaginary parts of current complex image estimate using weights from (iii): $m_{N L M}^{n}=N L M\left(m_{\text {data }}^{n}\right)$

(v) Update the image estimate: $m^{n+1}=m^{n}+$ $\alpha\left(m_{N L M}^{n}-m^{n}\right), \alpha$ is the step size.

Steps (ii)-(v) are repeated until the difference between new image estimate, $m^{n+1}$ and current image $m^{n}$ is below a certain threshold or a certain maximum number of iterations (500) is reached. The reconstruction parameters were chosen as $\operatorname{Win}(p)=$ $7 X 7, h=0.5 \sigma, N_{p}=N_{q}=5 X 5, \alpha=0.1, m^{0}$ was chosen as the inverse Fourier transform of undersampled data.

A similar type of approach but using a bilateral filter was proposed recently [20]. It was reported that using a bilateral filter fits in as a homotopic approximation of the $L_{0}$ norm and results in sparse solutions. The method was tested on simulated phantom and in-vivo MR images and results were similar to those obtained when using an $L_{1}$ norm/TV approach. The approach here is built on a more realistic NLM based model for MR images and results in improved reconstructions over L1 norm approach.

\subsection{Data acquisition}

Fully sampled Cartesian k-space data of T1-weighted breast images $(\mathrm{TR}=2.35 \mathrm{~ms}, \mathrm{TE}=0.99 \mathrm{~ms}$, flip angle $=10^{\circ}, 3 \mathrm{D}$ gradient echo saturation recovery sequence) and of the brain (TR=3730 ms, TE $=105 \mathrm{~ms}$, slice thickness $=5 \mathrm{~mm}, 2 \mathrm{D}$ spin echo sequence) were acquired on Siemens 1.5T scanners. The data was undersampled offline by a factor of two $(\mathrm{R}=2)$ to simulate an accelerated acquisition. A variable density random sampling scheme was used in which 13 contiguous central $\mathrm{k}$-space phase encoding lines were kept and remaining lines were discarded or kept in a random fashion. For dynamic breast data since the data was 3D k-space, a 1D Inverse Fourier Transform (IFT) was first applied and each slice and each time frame was processed separately.

\section{Results}

Figures 1 and 2 show the results on breast and brain datasets respectively. A relatively noisy coil was 
chosen for breast data in order test the method's robustness to reconstruction. Figures $1 \mathrm{c}$ and $2 \mathrm{c}$ show vastly improved quality over Figures $1 \mathrm{~b}$ and $2 \mathrm{~b}$ in terms of removing the artifacts. For comparison we show the images obtained from $\mathrm{R}=2$ data using the $L_{1}$ norm/TV approach [7-9]. Iterative gradient descent was used for TV reconstruction as described in [9]. While the images in Figures $1 \mathrm{~d}$ and $2 \mathrm{~d}$ have reduced artifacts, overall they have lower fidelity to images from fully sampled data and higher residual ghosting as compared to Figures $1 \mathrm{c}$ and $2 \mathrm{c}$ respectively. For example the smaller tumor in the breast image is better delineated with NLM approach and the difference image for NLM has less defined circumference of the breast as compared to TV method. Sulcal CSF structures in grey matter in the brain image are smudged and are blockier with TV reconstruction as shown in the zoomed images. The RMS error for the L1 recon was higher than that for NLM recon by $17 \%$ for breast image and by $21 \%$ for brain image. SNR index was also computed by dividing mean signal from a uniform region in the image with the standard deviation of noise computed from a region in the background. Same regions were used for all the methods. While the L1 method had higher SNR than original fully sampled image for breast data, NLM reconstruction had $22 \%$ higher SNR than the L1 approach. For the brain image NLM had $20 \%$ higher SNR than L1 reconstruction.
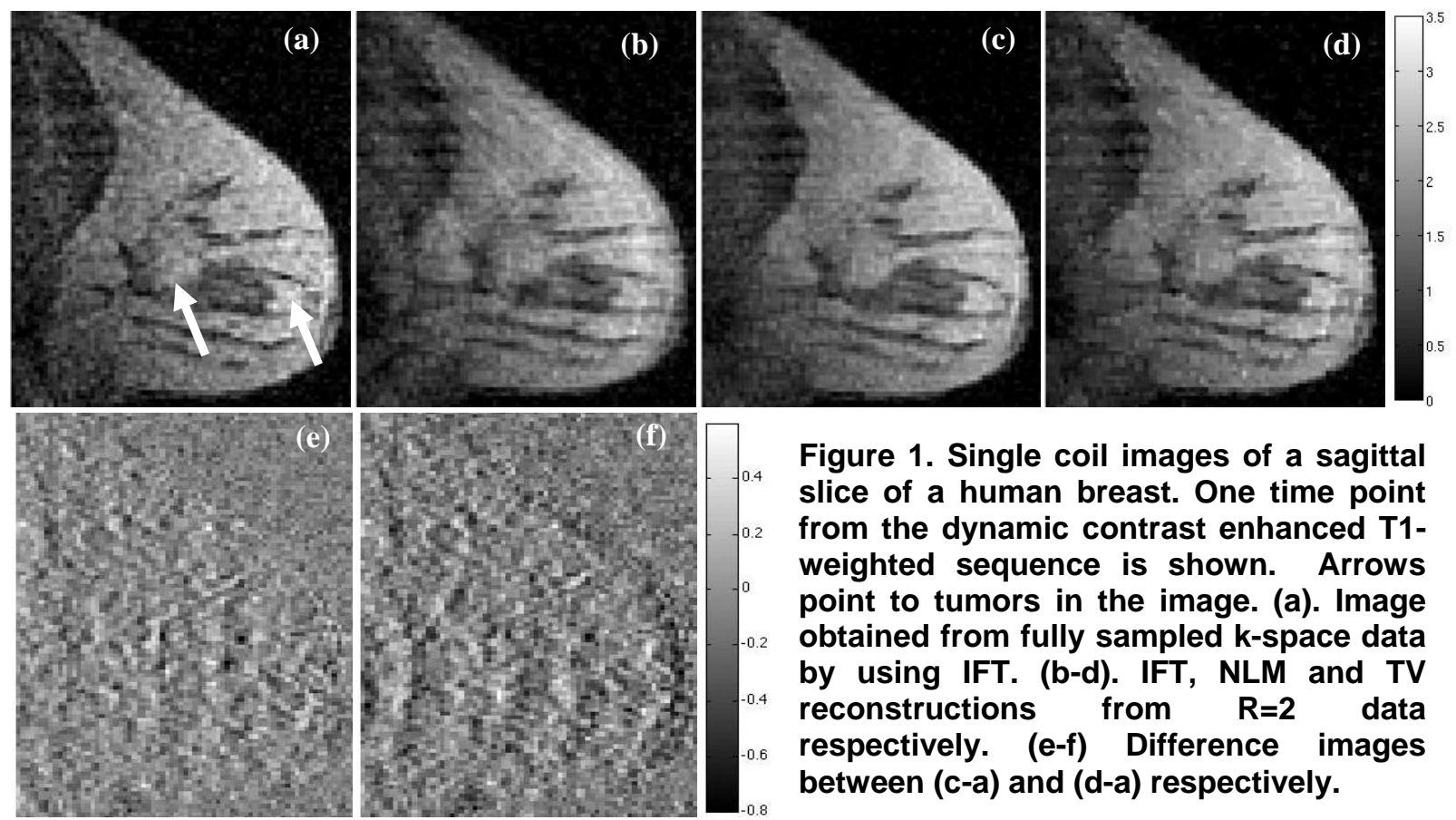

Figure 1. Single coil images of a sagittal slice of a human breast. One time point from the dynamic contrast enhanced T1weighted sequence is shown. Arrows point to tumors in the image. (a). Image obtained from fully sampled k-space data by using IFT. (b-d). IFT, NLM and TV reconstructions from $R=2$ data respectively. (e-f) Difference images between (c-a) and (d-a) respectively.
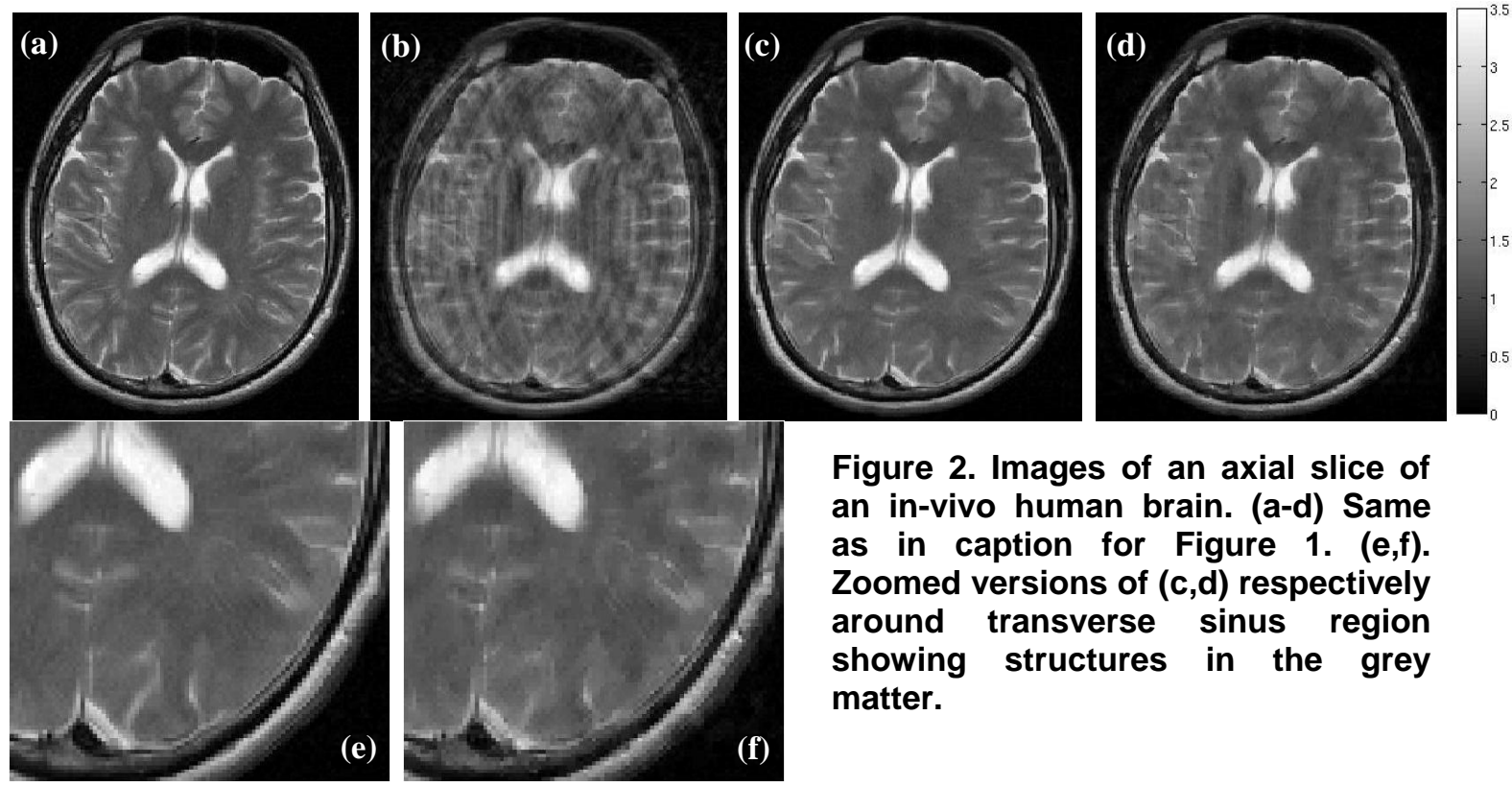

Figure 2. Images of an axial slice of an in-vivo human brain. (a-d) Same as in caption for Figure 1. (e,f). Zoomed versions of $(c, d)$ respectively around transverse sinus region showing structures in the grey matter. 


\section{Discussion}

The results show that the NLM framework can be used to improve the image reconstruction significantly when the data are undersampled. Unlike the linear approaches or the non-linear methods that use local information like signal intensity gradients, NLM uses information from a larger neighborhood and results in better artifact reduction.

\subsection{Parameter Choice}

As with most iterative reconstruction methods, choosing optimal parameters is important to obtain good results - choosing a large $h$ results in blurry images while the artifacts can be exacerbated when $h$ is too small. Parameters $\operatorname{Win}(p)$ and $N_{p}$ depend on image resolution and choosing a very large $\operatorname{Win}(p)$ may not be optimal as it results in using pixels that are not similar to pixel of interest even though their weights are small. Choosing a large $N_{p}$ results in finding fewer similar neighborhoods and can result in poor artifact suppression. We found that empirically determined set of parameters gave similar results for datasets within the same class.

\subsection{Computation Time}

The NLM approach is a computationally intensive algorithm but allows for parallel implementation on multi-core processors which can offer significant speed ups. For example with an efficient parallel implementation on a two core $2.5 \mathrm{Ghz}$ processor in Matlab, it took about 0.5 secs for steps (iii, iv) above for the breast data (104X80). Methods proposed in $[21,22]$ can also be used to further speed up and improve the results.

\section{Conclusion}

Non-local means offers a promising method to improve MR image reconstruction from undersampled $\mathrm{k}$-space data. The method has great potential to overcome limitations of traditional reconstruction methods for accelerating data acquisitions even when the original data is noisy.

Acknowledgements: We acknowledge Dr. Matthias Schabel for providing the breast data and Dr. Hee Kwon Song for help with brain data acquisition.

\section{References}

[1] B. Madore, G.H. Glover, N.J. Pelc. Unaliasing by fourier-encoding the overlaps using the temporal dimension (UNFOLD), applied to cardiac imaging and fMRI. Magn Reson Med. 42(5):813-28, 1999

[2] E.V.R. DiBella, Y.J. Wu, A.L. Alexander, D.L. Parker, D. Green, C.J. McGann. Comparison of temporal filtering methods for dynamic contrast MRI myocardial perfusion studies. Magn Reson Med 49:895-902,2003.

[3] J. Tsao, P. Boesiger, K.P. Pruessmann. k-t BLAST and k-t SENSE: Dynamic MRI with high frame rate exploiting spatiotemporal correlations. Magn Reson Med 50:1031$1042,2003$.

[4] L.I. Rudin, S. Osher, E. Fatemi. Nonlinear total variation based noise removal algorithms. Physica D, 60:259 $268,1992$.

[5] E. Candes, J. Romberg, T. Tao. Robust uncertainity principles: exact signal reconstruction from highly incomplete frequency information. IEEE Trans Information Theory ;52:489-509,2006.

[6] D.L. Donoho. Compressed sensing. IEEE Trans Info Theory; 52:1289-1306; 2006.

[7] K.T. Block, M. Uecker, J. Frahm. Undersampled radial MRI with multiple coils. Iterative image reconstruction using a total variation constraint. Magn Reson Med 57:1086-1098, 2007.

[8] M. Lustig, D. Donoho, J.M. Pauly. Sparse MRI: the application of compressed sensing for rapid MR imaging. Magn Reson Med; 58:1182-1195,2007.

[9] G. Adluru, C. Mc Gann, P. Speier, E.G. Kholmovski, A. Shaaban, E.V.R. DiBella. "Acquisition and reconstruction of undersampled radial data for myocardial perfusion MRI". J Magn Reson Imag 29:466-473, 2009.

[10] A. Buades, B. Coll, J. M. Morel. A Review of Image Denoising Algorithms, With a New One. Multiscale Model. Simul. 4(2), pp 490-530, 2005.

[11] S. Awate, R. Whitaker, "Image denoising with unsupervised information-theoretic adaptive filtering", IEEE Int Conf Computer Vision, pp. 44-51, 2005

[12] S. Awate, R. Whitaker, "Feature-preserving MRI denoising using a nonparametric, empirical-Bayes approach", IEEE Trans. Medical Imaging, 26(9), pp. 12421255, 2007.

[13] J.V. Manjón, J. Carbonell-Caballero, J.J. Lull, G. García-Martí, L. Martí-Bonmatí, and M. Robles, MRI denoising using Non-Local Means. Med. Image Analysis. 12(4), pp 514-523, 2008.

[14] P. Coupé, P. Yger, S. Prima, P. Hellier, C. Kervrann, C. Barillot. An Optimized Blockwise Non Local Means Denoising Filter for 3D Magnetic Resonance Images. IEEE Trans Medical Imaging, 27(4):425-441, 2008.

[15] J.V. Manjón, N.A. Thacker, J.J. Lull, G. Garcia-Martí, L. Marti-Bonmati, and M. Robles. Multicomponent MR Image Denoising. Int Journal Biomed imag, Article ID 756897, 10 pages, 2009.

[16] R.J. Marks II. Alternating Projections onto convex sets. Deconvolution of images and spectra ( $2^{\text {nd }}$ ed.), Academic Press, Inc. Pages: 476-501, 1996.

[17] E. Y. Sidky, X. Pan. Image reconstruction in circular cone-beam computed tomography by constrained, totalvariation minimization. Phys Med Biol 53, 4777-4807, 2008. [18] L. Chen, E.V.R. DiBella. POCS based reconstruction for dynamic MRI. Proc. ISMRM workshop on Data sampling and Image reconstruction, Sedona, 2009.

[19] M. Protter, M. Elad, H. Takeda, P. Milanfar. Generalizing the Non local-Means to Super-Resolution Reconstruction. IEEE Trans Image Proc. 18:36-51,2009.

[20] J. Trzasko, A. Manduca, E. Borisch. Robust Kernel Methods for Sparse MR Image Reconstruction. LNCS 4791:809-816, MICCAI, 2007.

[21] M. Mahmoudi and G. Sapiro, Fast image and video denoising via nonlocal means of similar neighborhoods," IEEE Signal Proc. Letters, vol. 12, no. 12, pp. 839-842, 2005.

[22] T. Tasdizen. Principal Neighborhood Dictionaries for Non-local Means Image Denoising. IEEE Trans Image Processing 18(12):2649-2660, 2009. 